\title{
Kulturelle Vielfalt in Österreichs Klassenzimmern: Welchen Beitrag kann eine Didaktik der Mehrkulturalität zur langfristig lernwirksamen Förderung von Lernenden im Englischunterricht der österreichischen Sekundarstufe I leisten?
}

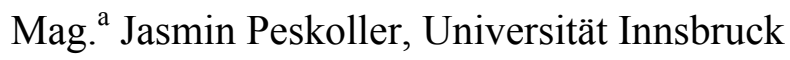

\section{Einleitung}

In diesem Beitrag wird ein Forschungsprojekt vorgestellt, das sich zum Ziel setzt, ein innovatives Unterrichtsdesign zu entwickeln, das auf die zunehmende kulturelle Vielfalt der Lernenden im Schulalltag eingeht und die Chancen nützt, die in dieser steigenden Mehrkulturalität stecken. Zunächst werden der Hintergrund der Studie sowie die schulischen Gegebenheiten mit Fokus auf den österreichischen Bildungskontext dargelegt. Im Anschluss wird der Stand der Forschung sowie Desiderata mit Schwerpunkt auf den Bereich der Sprachdidaktik angerissen und folglich ein Bezug $\mathrm{zu}$ den Sustainable Development Goals (SDGs) (siehe Rieckmann in diesem Band) hergestellt. Schließlich wird das Forschungsprojekt in seiner Konzeption präsentiert sowie dessen Zielsetzung der Anbahnung von mehrkulturellen Lernprozessen im Englischunterricht der Sekundarstufe I dargelegt. 


\section{Hintergrund}

In Zeiten von Globalisierung, Migration und Internationalisierung tritt täglich eine Vielzahl an Sprachen und Kulturen miteinander in Kontakt. Seit 2010 leben im deutschsprachigen Europa Menschen aus beinahe allen Regionen der Welt (Gogolin, 2016, S. 61). Mit dieser Entwicklung geht eine Zunahme der sprachlichen, kulturellen und sozialen Heterogenität der Bevölkerung einher (Sturm, 2016), die dazu führt, dass die Schule zu einem Ort der Mehrsprachigkeit und Mehrkulturalität wird (Bierwirth, Blell, \& Fuchs, 2017). In Bezug auf die weltweit zunehmende Vielfalt und Heterogenität im Bildungsbereich bekräftigt der australische Sprachdidaktiker Joseph Lo Bianco: "[T]he strongest indicator of the transformed realities of contemporary education in a globalised world is the depth of cultural, racial and linguistic diversity in schools" (Lo Bianco, 2009, S. 113).

Diese steigende Diversität in Gesellschaft und Schule spiegelt sich auch in Österreichs Klassenzimmern wider. Statistische Kennzahlen zeigen, dass in den Jahren 2011 bis 2016 der Prozentsatz an Menschen, die in Österreich leben, aber außerhalb Österreichs geboren wurden, von 15,7\% auf 18,8\% gestiegen ist (Statistik Austria, 2018a). Außerdem gaben in der letzten Volkszählung insgesamt 2,02 Millionen Menschen in Österreich an, einen Migrationshintergrund zu haben, was 23,3\% der Gesamtbevölkerung Österreichs entspricht (Statistik Austria, 2019). Die folgenden Abbildungen visualisieren das Ausmaß und die Entwicklung von Mehrsprachigkeit und Mehrkulturalität im österreichischen Bildungssystem anhand von statistischen Daten aus den Schuljahren 2017/18 sowie 2007/08 (Statistik Austria, 2018b). An dieser Stelle ist anzumerken, dass sich aus dem in den Abbildungen verwendeten Begriff ,Erstsprache nicht Deutsch` eine Form von Migrationsgeschichte deduzieren lässt und somit sprachliche Vielfalt als Indikator für kulturelle Vielfalt dient.

Kapelari, Suzanne (Hg.), Vierte „Tagung der Fachdidaktik“ 2019:

„Interdisziplinäre fachdidaktische Diskurse zur Bildung für nachhaltige Entwicklung“

(C) 2020 innsbruck university press, ISBN 978-3-99106-019-2, DOI 10.15203/99106-019-2 


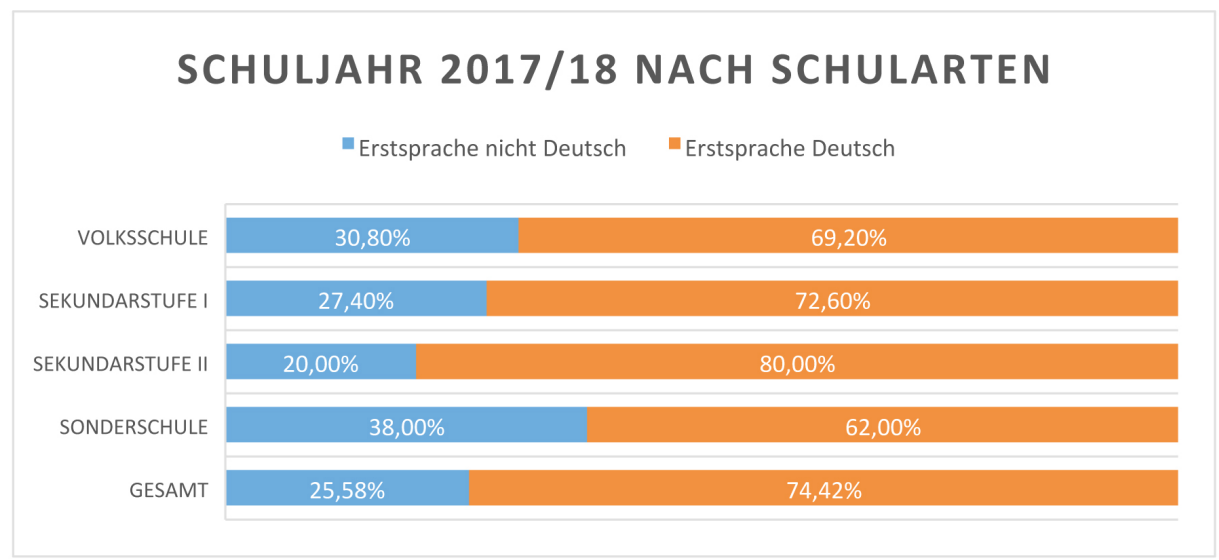

Abb. 1: Erstsprachen der Lernenden im Schuljahr 2017/18 nach Schularten (Eigene Darstellung J.P.)

Abbildung 1 verdeutlicht, dass im Schuljahr 2017/18 exakt ein Fünftel der SchülerInnen in der Sekundarstufe II angaben, eine nicht deutsche Erstsprache zu sprechen. Dieser Wert lag im Vergleich dazu in der Sekundarstufe I bei 27,4\%. Aufbauend auf diese Visualisierung bietet Abbildung 2 einen genaueren Einblick in die sprachlichen Gegebenheiten der SchülerInnen in der Sekundarstufe I, indem diese nach Schultypen aufgespaltet betrachtet wird. 


\section{SCHULJAHR 2017/18 SEKUNDARSTUFE I}

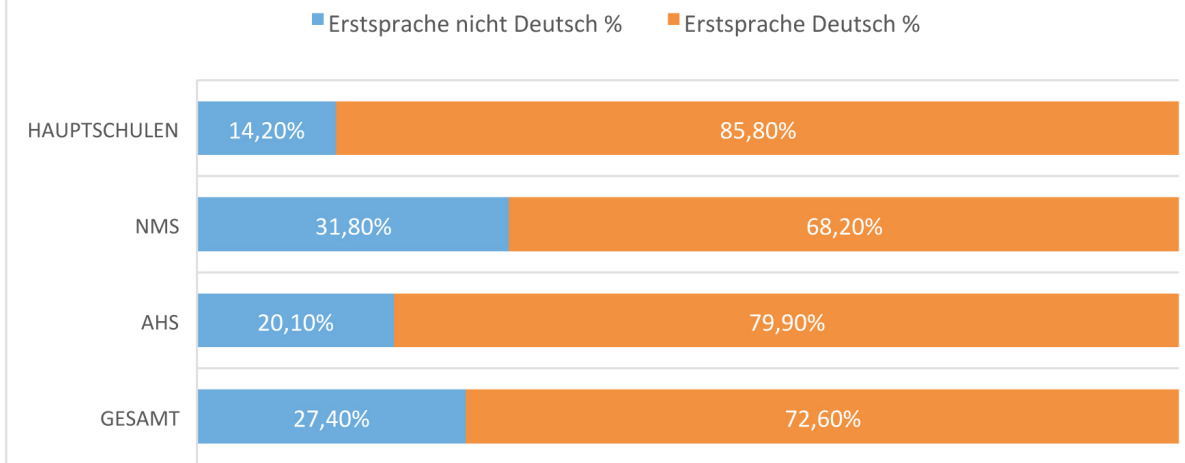

Abb. 2: Erstsprachen der Lernenden im Schuljahr 2017/18 in der Sekundarstufe I (Eigene Darstellung J.P.)

An Abbildung 2 lässt sich erkennen, dass im Schuljahr 2017/18 an Neuen Mittelschulen (NMS) der Anteil an Lernenden mit anderen Erstsprachen als Deutsch mit 31,8\% deutlich höher lag als an AHS-Schulen mit 20,1\% (Statistik Austria, 2018b). Abbildung 3 bietet nun einen 10-Jahres-Vergleich zum Anteil der Lernenden mit anderer Erstsprache als Deutsch, indem die Schuljahre 2017/18 und 2007/08 kontrastiert werden.

Es ist erkenntlich, dass im Schuljahr 2017/18 insgesamt mehr als ein Viertel aller Lernenden in Österreich angaben, eine andere Erstsprache als Deutsch zu sprechen. 10 Jahre zuvor lag dieser Prozentsatz erst bei rund 15,9\%, was einer Zunahme von über $60 \%$ entspricht. 


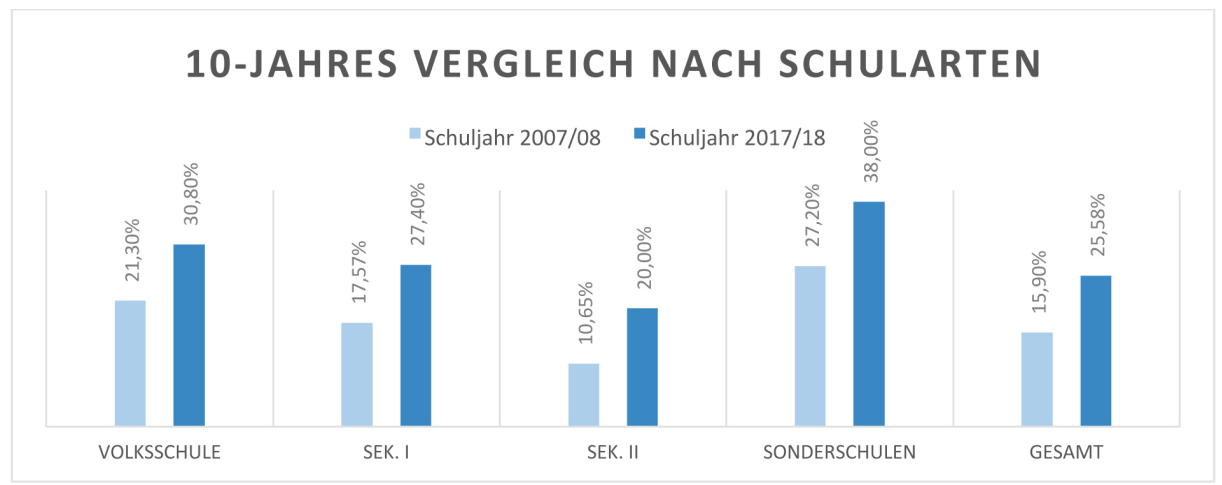

Abb. 3: Anteil Lernender mit Erstsprache nicht Deutsch im Schuljahr 2007/2008 und 2017/2018 (Eigene Darstellung J.P.)

\section{Forschungsstand und Desiderata}

In diesem Abschnitt soll nun der Stand der Forschung sowie Problemstellungen und Desiderata im Forschungsfeld der kulturellen Diversität im (Sprach-) Unterricht mit Fokus auf das österreichische Schulsystem beleuchtet werden.

Aufgrund der in Abschnitt 2 dargestellten Veränderungen in der SchülerInnenpopulation ergibt sich die Notwendigkeit, existierende Lehr- und Lernansätze entsprechend anzupassen und effektive Unterrichtsdesigns zu entwickeln, die die LernerInnen auf ein Leben in einer mehrsprachigen und mehrkulturellen Gesellschaft vorbereiten (Anton, 2017; Brunsmeier, 2016; Dausend, 2014; Delanoy, 2014a). Da Sprache und Kultur eng miteinander verzahnt sind (Freitag-Hild, 2018) und jeder Sprachunterricht gleichzeitig auch Kulturunterricht ist (Kramsch, 2006, S. 8), kommt der Sprachbildung in Bezug auf kulturelles Lernen eine besondere Rolle zu (Hu \& Byram, 2009). Da Sprache nicht nur Teil von Kultur ist, sondern diese gleichzeitig auch widerspiegelt und begründet, muss für erfolgreiches Kommunizieren Sprachverwendung eng mit kulturell angemessenem Verhalten gedacht werden (Blell \& Doff, 2014a, S. 78). Aufgrund ihrer Funktion als lingua franca, deren 
Reichweite historisch einzigartig ist (Baker, 2015, S. 9), hat die englische Sprache in diesem Zusammenhang einen speziellen Stellenwert, da sie die Grundlage des internationalen Austauschs zwischen Menschen unterschiedlicher kultureller Hintergründe darstellt (Brunsmeier, 2016, S. 13). Folglich wird das Einbeziehen einer Vielzahl an Kulturen in Lehr-Lernprozesse propagiert (Schlaak, 2015), da auf diese Weise die gesellschaftliche Realität im Klassenzimmer authentisch abgebildet und der Unterricht somit der zunehmenden sprachlichen und kulturellen Vielfalt der Lernenden gerecht werden kann (Burwitz-Melzer, 2017; Reimann, 2017; Schäfer, 2016, 2017a). In diesem Zusammenhang bekräftigt Doff (2016, S. 4), dass die Konzeption des Fremdsprachenunterrichts im Allgemeinen am stärksten durch sprachliche und kulturelle Heterogenität beeinflusst werden.

Als Folge dieser Entwicklungen und dem besonderen Stellenwert von Englisch als lingua franca in der interkulturellen Kommunikation (Baker, 2015) hat sich die Förderung der sogenannten interkulturellen (kommunikativen) Kompetenz (IKK) als übergeordnetes Ziel des Fremdsprachenunterrichts etabliert (Blell \& Doff, 2014b). Die Entwicklung von IKK wurde auch im grundlegenden Bildungsdokument des europäischen Sprachunterrichts, dem Gemeinsamen Europäischen Referenzrahmen für Sprachen (GERS), manifestiert (Council of Europe, 2001). Zudem stellt interkulturelles Lernen ein grundlegendes Bildungsziel und didaktisches Prinzip in den österreichischen Lehrplänen dar (BMBWK / Bundesministerium für Bildung, Wissenschaft und Kunst: Österreichischer Lehrplan AHS Unterstufe Lebende Fremdsprachen, 2006), dessen Bedeutsamkeit für schulische Lernprozesse in einem Grundsatzerlass im Jahr 2017 erneut bekräftigt wurde (BMB / Bundesministerium für Bildung: Interkulturelle Bildung: Grundsatzerlass, 2017).

Die für diesen Beitrag grundlegenden Begriffe des inter-, trans- und mehrkulturellen Lernens sollen nun skizziert werden. Vereinfacht dargestellt bezieht sich der Begriff interkulturell auf Gegebenheiten, in welchen Aspekte oder Menschen kulturell getrennt und verglichen werden und eine Gegenüberstellung zwischen „Selbst“ und „Anderem“ bzw. „Fremdem“

Kapelari, Suzanne (Hg.), Vierte „Tagung der Fachdidaktik“ 2019:

„Interdisziplinäre fachdidaktische Diskurse zur Bildung für nachhaltige Entwicklung“

(C) 2020 innsbruck university press, ISBN 978-3-99106-019-2, DOI 10.15203/99106-019-2 
stattfindet (Fäcke \& Meißner, 2019, S. 5). Im Sprachunterricht wird damit die Kompetenz verstanden, die Erstkultur mit der Zielkultur in Zusammenhang zu bringen und über Unterschiede zu reflektieren (Council of Europe, 2001, S. 104-105). Im Gegensatz dazu versucht das seit der Jahrtausendwende bestehende Konzept des transkulturellen Lernens (Welsch, 1999) diese Dichotomie aufzuheben und den Fokus auf vereinende, gemeinsame Aspekte sowie Hybridisierung zu legen (Fäcke \& Meißner, 2019, S. 5). Um kulturelle Lernprozesse im Sprachunterricht zu untersuchen entwickelte Michael Byram mit seinem Modell der IKK (Byram, 1997) eine Operationalisierung des interkulturellen Lernens. Obwohl das Modell bis heute als Grundlage zahlreicher Studien dient, haben konzeptuelle und terminologische Debatten zur Erweiterung bzw. Ablöse des Konstrukts des interkulturellen Lernens durch das transkulturelle Lernen zugenommen (Delanoy, 2014b; Freitag-Hild, 2018; Reimann, 2015). Folglich stellt der Begriff der Mehrkulturalität eine Klammer für inter- und transkulturelle Lernprozesse dar (Blell \& Doff, 2014b, S. 2) und setzt sich zum Ziel, kulturelle Hintergründe der SchülerInnen stärker in das Unterrichtsgeschehen miteinzubinden, wie es von zahlreichen AutorInnen gefordert wird (Bierwirth et al., 2017; Burwitz-Melzer, 2017; Doff, 2016; Reimann, 2017; Schlaak, 2015).

Neben einigen Handlungsempfehlungen zur Anbahnung von inter- und transkulturellen, demnach mehrkulturellen, Ansätzen im Sprachunterricht und trotz dessen mehrfach akzentuierter Bedeutung für Lernprozessen, lässt sich ein deutlicher Mangel an empirischer Forschung konstatieren (Anton, 2017; Brunsmeier, 2016; Schäfer, 2016; Trautmann, 2010). Eine der wenigen kürzlichen Forschungsarbeiten, die sich um die Schließung der Lücke an Empirie in Zusammenhang mit kulturellen Lernprozessen im Englischunterricht bemüht, sind Brunsmeier (2016) und Schäfer (2016). In ihrer Studie entwickelte Brunsmeier (2016) Aufgaben anhand von theoriegeleiteten Könnensbeschreibungen für den Englischunterricht der Primarstufe und erprobte empirisch, ob diese zur Förderung der interkulturellen kommunikativen Kompetenz der Lernenden beitragen. Im Englischunterricht der Sekundarstufe I beleuchtete Schäfer (2016) hingegen Möglichkeiten der Anbahnung kulturellen Lernens im

Kapelari, Suzanne (Hg.), Vierte „Tagung der Fachdidaktik“ 2019:

„Interdisziplinäre fachdidaktische Diskurse zur Bildung für nachhaltige Entwicklung“

(C) 2020 innsbruck university press, ISBN 978-3-99106-019-2, DOI 10.15203/99106-019-2 
Kontext heterogener Lerngruppen. Sie folgert aus ihrer design-based research Studie, dass die kulturelle Vielfalt der SchülerInnen über die Interaktion von und über verschiedene Sichtweisen und Deutungsmuster in den Englischunterricht miteinbezogen werden kann.

Neben diesen vereinzelten Forschungsarbeiten beklagt Doff (2016, S. 4) ein besonderes Defizit an Forschung im Englischunterricht der Sekundarstufe I. Des Weiteren konnte eine vorherrschende Unsicherheit der Lehrenden in Bezug auf den Umgang mit kultureller Vielfalt im Unterricht (Chilla \& Vogt, 2017) sowie bezüglich der Implementierung von inter- und transkulturellen Lernprozessen festgestellt werden (Brunsmeier, 2016). Obwohl das Bewusstsein der Lehrpersonen über das Bildungsziel der IKK nachgewiesen werden konnte (Brunsmeier, 2016, S. 90), führt diese Unsicherheit häufig zu einer Reduzierung der komplexen Konstrukte auf die Vermittlung von Landeskunde (Grünewald, 2012, S. 55),

Schließlich wird die Notwendigkeit deutlich, geeignete Aufgabenstellungen für den Sprachunterricht zu entwickeln, deren Potenzial zur Anbahnung mehrkultureller Lernprozesse zu untersuchen und Implikationen für schulische Lehr-Lernprozesse abzuleiten, durch die Lehrpersonen in der Implementierung des Konstrukts unterstützt werden können (Anton, 2017; Bär, 2017; Brunsmeier, 2016; Göbel, Lewandowska, \& Diehr, 2017; Schäfer, 2017b).

\section{Mehrkulturalität und Bildung für nachhaltige Entwicklung}

In diesem Abschnitt soll der Zusammenhang sowie mögliche Anknüpfungspunkte zwischen dem Forschungsfeld der kulturellen Vielfalt im (Sprach)Unterricht sowie der Bildung für nachhaltige Entwicklung herausgearbeitet werden.

In Bezug auf kulturelle Vielfalt und Bildungserfolg hält die Erziehungswissenschaftlerin Ingrid Gogolin (2016, S. 62) fest, dass die Forschung zeigt, dass

Kapelari, Suzanne (Hg.), Vierte „Tagung der Fachdidaktik“ 2019:

„Interdisziplinäre fachdidaktische Diskurse zur Bildung für nachhaltige Entwicklung“

(C) 2020 innsbruck university press, ISBN 978-3-99106-019-2, DOI 10.15203/99106-019-2 
Lernende mit Migrationshintergrund die Schullaufbahn durchschnittlich deutlich weniger erfolgreich durchlaufen als Gleichaltrige ohne Migrationshintergrund. Sie kritisiert zudem den Mangel an empirischer Forschung, die die Bedeutung der Unterrichtsgestaltung zum potenziellen Entgegenwirken dieser Differenzen untersucht. Diesbezüglich wird von Bierwirth, Blell und Fuchs argumentiert:

„Das Primat, allen Schüler*innen gleiche Chancen auf Bildung bzw. eine maximale Bildungsteilhabe zu gewähren, muss stärker in den Blick schulischen Alltags und sämtlicher Reformbemühungen im Bildungsbereich genommen werden. Eine Pädagogik der Vielfalt, oder auch der sogenannte diversity-Ansatz (Anerkennung der Vielfalt), wird auf vielen gesellschaftlichen Ebenen diskutiert - darunter auch der Bildungsbereich und sollte ein wesentliches Merkmal unserer Gesellschaft sein.“ (Bierwirth et al., 2017)

Diese Forderung nach Chancengleichheit in der Bildung findet sich auch in den von den Vereinten Nationen im Jahr 2015 formulierten politischen Zielen für nachhaltige Entwicklung, den so genannten Sustainable Development Goals (SDGs, siehe Rieckmann in diesem Band), wieder. Der Bildungsbereich soll hierbei bestmöglich einen Beitrag zu jedem formulierten Ziel leisten (United Nations, 2019a).

Schnittstellen zwischen den SDGs und dem Forschungskontext der kulturellen Vielfalt im (Sprach-)Unterricht sind der Bereich 4 (Quality Education) sowie SDG 10 (Reduced Inequalities). Letzteres beschreibt, dass es grundsätzlich universeller politischer Bestimmungen bedarf, um verstärkt auf die Bedürfnisse der benachteiligten und marginalisierten Bevölkerung $\mathrm{zu}$ achten (United Nations, 2019b). Weiters führt der Bereich Nummer 4, der sich konkret auf den Bildungsbereich bezieht, an, dass eine Verbesserung der Bildungsgegebenheiten durch eine adäquate Ausbildung der Lehrkräfte, vor allem im Umgang mit Minderheiten und Heterogenität, erreicht werden kann (United Nations, 2019c). Die internationale Bildungskampagne ,Bildung für nachhaltige Entwicklung hält folglich fest: 
„Kulturkonflikte treten in verschiedenen Gesellschaftsbereichen auf, so auch in Kontexten der Schule. Die Bereitschaft zum Dialog ist eine wichtige Voraussetzung, um den Mehrwert kultureller Diversität erfahren zu können. In Schulen bietet sich insbesondere die Gelegenheit - etwa durch Gruppenarbeit im Besonderen und den Klassenverband im Allgemeinen soziale Kompetenzen zu erlernen.“(UNESCO-Weltaktionsprogramm, 2019)

In ähnlicher Formulierung findet sich die obige Aussage auch in dem (Sprach-) Unterricht zugrunde liegenden, bildungspolitischen Dokumenten wie dem GERS (Council of Europe, 2001, 2018) oder den österreichischen Lehrplänen wieder. In den Schulcurricula ist interkulturelles Lernen als ein Bildungsprinzip verankert (BMB / Bundesministerium für Bildung: Interkulturelle Bildung: Grundsatzerlass, 2017) und $\mathrm{zu}$ vermittelnde Werte wie Gleichberechtigung, Offenheit und Respekt werden besonders hervorgehoben (BMBWK / Bundesministerium für Bildung, Wissenschaft und Kunst: Österreichischer Lehrplan AHS Unterstufe Lebende Fremdsprachen, 2006). Somit wird deutlich, dass eine erhöhte Chancengleichheit im Bildungserfolg (SDG 10) und eine gesteigerte Qualität des Lehrens und Lernens (SDG 4) vor allem durch eine verstärkte Schulung der Lehrpersonen, speziell in Hinblick auf den effizienten und sensiblen Umgang mit kultureller Vielfalt, erreicht werden kann.

Es ist deshalb essentiell zu erkennen, dass Bildung niemals als kulturneutral gedacht werden darf, Lernprozesse nicht in einem Vakuum stattfinden und weder Lehrende noch Lernende frei von kulturellen Einflüssen sind. Es muss Ziel nachhaltiger Bildung sein, ein Bewusstsein und eine Wertschätzung für kulturelle Vielfalt zu schaffen und dadurch Lernende in der Entwicklung ihrer kulturell verankerten Fähigkeiten zu unterstützen (UNESCO, 2009, S. 97).

Eine weitere Schnittstelle zwischen Bildung für nachhaltige Entwicklung und Mehrkulturalität im (Sprach-)Unterricht stellt der Kompetenzbegriff dar. Wie in Abschnitt 3 erläutert bietet Byrams 5-dimensionales Modell der interkulturellen kommunikativen Kompetenz (Byram, 1997), welche die Fähigkeit meint, „relevante Einstellungen, Fertigkeiten, Wissen und kritisches Verständnis zu nutzen, um angemessen auf Herausforderungen und Gelegenheiten in der

Kapelari, Suzanne (Hg.), Vierte „Tagung der Fachdidaktik“ 2019:

„Interdisziplinäre fachdidaktische Diskurse zur Bildung für nachhaltige Entwicklung“

(C) 2020 innsbruck university press, ISBN 978-3-99106-019-2, DOI 10.15203/99106-019-2 
Interaktion mit Vertretern einer anderen kulturellen Gruppe zu reagieren“ (Byram, 2019, S. 252), eine Grundlage für Forschungsarbeiten in der Sprachendidaktik. Auf dem Gebiet der Nachhaltigkeitsforschung wird in ähnlicher Form die so genannte interpersonal competence als eine von fünf definierten Kernkompetenzen von sustainability genannt. Interpersonal competence beschreibt die Fähigkeit, die Vielfalt an Kulturen, sozialen Gruppen, Gemeinschaften und Individuen zu verstehen, aufzugreifen und $\mathrm{zu}$ fördern. Da für diesen Kompetenzbereich Aspekte wie pluralistisches und transkulturelles Denken relevant sind (Wiek, Withycombe, \& Redman, 2011, S. 211), ergeben sich hier Synergien zwischen dem Forschungsfeld der Mehrkulturalität und dem der Nachhaltigkeit, deren Potenzial in künftigen Studien vermehrt ausgeschöpft werden kann. An dieser Stelle ist positiv zu vermerken, dass der Begriff des transkulturellen Lernens bereits Einzug in den Kompetenzdiskurs findet.

In Bezug auf Bildungsgerechtigkeit und Mehrkulturalität plädiert Doff (2016, S. 4) für die Generierung von sowohl fachspezifischen als auch interdisziplinär ausgerichteten, empirisch überprüften Methoden und Ansätzen, die das Potenzial der lebensweltlichen Mehrkulturalität der Lernenden in der Schule nutzen und aktiv in das Unterrichtsgeschehen einbinden. Gogolin (2016, S. 64) vermerkt diesbezüglich in letzter Zeit eine Zunahme der Forschungsaktivität in Hinblick auf die Gestaltung von Lehr-Lernprozessen im Bildungsbereich zur Nutzung der Chancen von sprachlicher und kultureller Heterogenität für das schulische Lernen.

\section{Forschungsprojekt}

In Anbetracht der zunehmenden kulturellen Vielfalt im österreichischen Bildungssystem (Statistik Austria, 2018b) und des bestehenden Mangels an Forschung und empirischen Daten zur Implementierung von mehrkulturellen Lernprozessen setzt sich die Forschungsarbeit zum Ziel, an einige der aufgezeigten Desiderate anzuknüpfen. Folglich ist eine empirische Studie in 
Planung, in welcher ein theoriebasiertes und praxisrelevantes methodisches Design für den Englischunterricht entwickelt, implementiert und empirisch evaluiert wird. Dieses Unterrichtsdesign bezieht im Sinne der Didaktik der Mehrkulturalität (Reimann, 2017) die kulturellen Gegebenheiten der Lernenden mit ein und leistet somit einen Beitrag in der Anbahnung von SDG 4 und 10 (United Nations, 2019a). Die Studie ist in der österreichischen Sekundarstufe I, konkret in Englischklassen der Neuen Mittelschule (NMS), verortet, da in diesem Schultyp das Ausmaß an sprachlicher und somit kultureller Vielfalt der Lernenden im Schuljahr 2017/18 besonders hoch war (siehe Abschnitt 2).

Die folgenden Fragestellungen sind für die Forschungsarbeit wegweisend:

Forschungsfrage 1: Wie werden mehrkulturelle Lernprozesse von Lehrpersonen im Englischunterricht der österreichischen Sekundarstufe I angebahnt?

Forschungsfrage 2: Welche Herausforderungen entstehen in Verbindung mit der Anbahnung von mehrkulturellen Lernprozessen im Englischunterricht der österreichischen Sekundarstufe I?

Forschungsfrage 3: Auf welche Art und in welchem Umfang werden mehrkulturelle Lernprozesse in den Aufgabenstellungen der Lehrwerke für den Englischunterricht der österreichischen Sekundarstufe I angebahnt?

Forschungsfrage 4: Welche Dimensionen des mehrkulturellen Lernens können durch Aufgabenorientierung im Englischunterricht der österreichischen Sekundarstufe I angebahnt werden?

Abbildung 4 visualisiert die Struktur des Forschungsprojekts, das durch Anwendung von sowohl qualitativen als auch quantitativen Elementen als

Kapelari, Suzanne (Hg.), Vierte „Tagung der Fachdidaktik“ 2019:

„Interdisziplinäre fachdidaktische Diskurse zur Bildung für nachhaltige Entwicklung“

(C) 2020 innsbruck university press, ISBN 978-3-99106-019-2, DOI 10.15203/99106-019-2 
mixed-methods Design (Kuckartz, 2014) konzipiert ist. Pro Abschnitt gibt die Abbildung zudem Aufschluss über die jeweilige Erhebungsart und das Sample und zeigt die Verbindung zu den obigen Forschungsfragen.

\section{TeIL 1: Bedarfsanalyse \& STATUS-Quo ERHEbung}

\subsection{ONLINE BEFRAGUNG (FORSCHUNGSFRAGE 1, 2)}

$\checkmark$ Quantitativ

$\checkmark$ Sample: 100 TeilnehmerInnen; Englisch Lehrpersonen in der österreichischen

Sekundarstufe I

\subsection{LEHRWERKSANALYSE (FORSCHUNGSFRAGE 3)}

$\checkmark$ Qualitativ \& Quantitativ

$\checkmark$ Korpus: 12 Englisch Lehrwerke für die österreichische Sekundarstufe I

(student's books, Arbeitsbücher und Lehrerhandbücher)

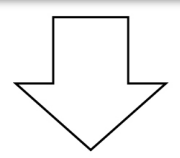

TEIL 2: DESIGN-BASED RESEARCH STUDIE (DBR)

Iterativ: Design, Versuch, Evaluierung, Re-design (FORSCHUNGSFRAGE 4)

\subsection{DBR-ZYKLUS I}

$\checkmark$ Qualitativ \& Quantitativ

$\checkmark$ Sample: Zwei Englischklassen der neuen Mittelschule (NMS), vier Lehrpersonen, ca. 35 Lernende

\subsection{DBR-ZYKLUS II}

$\checkmark$ Qualitativ \& Quantitativ

$\checkmark$ Sample: Zwei Englischklassen der neuen Mittelschule (NMS), vier Lehrpersonen, ca. 35 Lernende

Abb. 4: Forschungsdesign (Eigene Darstellung J.P.) 
Wie in Abbildung 4 ersichtlich ist, wird im ersten Teil der Studie eine StatusQuo Erhebung zum Status und zur Umsetzung des Bildungsziels des interkulturellen Lernens im Englischunterricht der Sekundarstufe I durchgeführt. Anhand dieser sollen Herausforderungen und Förderbedarf in Zusammenhang mit der Anbahnung von inter-, trans- und somit mehrkulturellen Lernprozessen aufgezeigt werden. Im anschließenden zweiten Teil der Forschungsarbeit wird eine design-based research Studie (DBR) (Euler, 2014) durchgeführt. Diese setzt sich zum Ziel, ein theoriebasiertes, praxisrelevantes Unterrichtsdesign nach Vorbild des aufgabenorientierten Sprachunterrichts iterativ zu entwickeln, zu erproben und schließlich zu evaluieren, inwieweit mehrkulturelle Lernprozesse im Englischunterricht der Sekundarstufe I angebahnt werden können. Durch das dabei entstehende, empirisch fundierte Unterrichtsmodell wird ein erster methodischer Rahmen zur Anbahnung von mehrkulturellen Lernprozessen im Englischunterricht geschaffen, der eine wertvolle Unterstützung für Lehrpersonen im Umgang mit kultureller Vielfalt im Unterricht darstellen kann.

\section{Zusammenfassung und Ausblick}

In diesem Beitrag wurde ein Forschungsprojekt vorgestellt, welches durch die Anbahnung von mehrkulturellen Lernprozessen im Englischunterricht der österreichischen Sekundarstufe I versucht, die Chancen der zunehmenden kulturellen Vielfalt der Lernenden aktiv in das Unterrichtsgeschehen miteinzubinden. Durch das entwickelte Unterrichtsdesign können Lehrpersonen sowohl in der Umsetzung von verankerten Bildungszielen als auch im Umgang mit kultureller Diversität im Klassenzimmer unterstützt werden. Es wurde erläutert, dass hier Überschneidungen mit den Grundanliegen der Bildung für Nachhaltige Entwicklung sowie den Sustainable Development Goals vorliegen. Ebenso zeigen sich in verwendeten Kompetenzmodellen der Sprach- sowie der Nachhaltigkeitsforschung Synergien und mögliche Ausrichtungen für künftige Forschungsarbeiten.

Kapelari, Suzanne (Hg.), Vierte ,Tagung der Fachdidaktik“ 2019:

„Interdisziplinäre fachdidaktische Diskurse zur Bildung für nachhaltige Entwicklung“

(C) 2020 innsbruck university press, ISBN 978-3-99106-019-2, DOI 10.15203/99106-019-2 
Abschließend ist festzuhalten, dass sprachliche und kulturelle Diversität in Gesellschaft und Bildung schon längst kein Novum mehr darstellen und aufgrund von Globalisierung und Migrationsbewegungen weiter zunehmen werden (Gogolin, 2016). Es ist deshalb von entscheidender Bedeutung, auf diese sich verändernden Gegebenheiten auch im Bildungskontext zu reagieren und folglich bestehende Unterrichtsansätze und -methoden zu überdenken und entsprechend zu adaptieren. Eine zeitgemäße Pädagogik und Didaktik muss sich zunehmend in Bezug auf mehrkulturelles Lernen reflektieren und hinterfragen, ob möglichst alle Lernenden von den Unterrichtsinhalten und -methoden angesprochen werden. Die kulturelle Vielfalt wird jedoch im Unterricht vermehrt als Herausforderung anstatt als Chance für das Lehren und Lernen wahrgenommen. Forschungsarbeiten könnten anhand entsprechender Erkenntnisse die Bedeutsamkeit der Thematik hervorheben und Lehrpersonen in Bezug auf den effektiven Umgang mit kultureller Vielfalt sensibilisieren. Die vorgestellte Studie stellt eine Möglichkeit dar, anhand von mehrkulturellen Lernprozessen der kulturellen Vielfalt der Lernenden gerecht zu werden und gleichzeitig alle SchülerInnen auf ein Leben in einer mehrsprachigen und mehrkulturellen Gesellschaft vorzubereiten.

\section{Literatur}

Anton, D. (2017). Inter- und transkulturelles Lernen im Englischunterricht: Eine didaktische Analyse einschlägiger Lehrbücher. Heidelberg: Universitätsverlag Winter.

Baker, W. (2015). Culture and identity through English as a Lingua Franca: Rethinking concepts and goals in intercultural communication. Developments in English as a Lingua Franca: Vol. 8. Berlin, Boston: De Gruyter Mouton. https://doi.org/10.1515/9781501502149

Bär, M. (2017). Auf dem Weg zur inklusiven Schule: Mögliche Implikationen aus fremdsprachendidaktischer Perspektive. In E. Burwitz-Melzer, F. G. Königs, C. Riemer, \& L. Schmelter (Eds.), Giessener Beiträge zur Fremdsprachendidaktik. Inklusion, Diversität und das Lehren und Lernen fremder Sprachen: Arbeitspapiere der 37. Frühjahrskonferenz zur Erforschung des Fremdsprachenunterrichts (pp. 10-20). Tübingen: Narr Francke Attempto.

Kapelari, Suzanne (Hg.), Vierte „Tagung der Fachdidaktik“ 2019:

„Interdisziplinäre fachdidaktische Diskurse zur Bildung für nachhaltige Entwicklung“

(C) 2020 innsbruck university press, ISBN 978-3-99106-019-2, DOI 10.15203/99106-019-2 
Bierwirth, A., Blell, G., \& Fuchs, S. (2017). Wie divers ist Englischlernen?: Konzepte in Forschung und Praxis zum inklusiven Englischunterricht. Zeitschrift Für Inklusion. (3). Retrieved from https://www.inklusion-online.net/index.php/inklusion-online/article/view/447

Blell, G., \& Doff, S. (2014a). It takes more than two for this tango: Moving beyond the self/other-binary in teaching about culture in the global EFL-classroom. Zeitschrift Für Interkulturellen Fremdsprachenunterricht, 19(1), 77-96.

Blell, G., \& Doff, S. (2014b). Mehrsprachigkeit und Mehrkulturalität: Einführung in das Thema. Zeitschrift Für Interkulturellen Fremdsprachenunterricht, 19(1), 1-7.

BMB / Bundesministerium für Bildung: Interkulturelle Bildung: Grundsatzerlass (2017).

BMBWK / Bundesministerium für Bildung, Wissenschaft und Kunst: Österreichischer Lehrplan AHS Unterstufe Lebende Fremdsprachen (2006).

Brunsmeier, S. (2016). Interkulturelle Kommunikative Kompetenz im Englischunterricht der Grundschule: Grundlagen, Erfahrungen, Perspektiven. Tübingen: Narr.

Burwitz-Melzer, E. (2017),Same same but different': Inklusion, Heterogenität und Diversität im Englischunterricht. In E. Burwitz-Melzer, F. G. Königs, C. Riemer, \& L. Schmelter (Eds.), Giessener Beiträge zur Fremdsprachendidaktik. Inklusion, Diversität und das Lehren und Lernen fremder Sprachen: Arbeitspapiere der 37. Frühjahrskonferenz zur Erforschung des Fremdsprachenunterrichts (pp. 31-42). Tübingen: Narr Francke Attempto.

Byram, M. (1997). Teaching and Assessing Intercultural Communicative Competence. Clevedon: Multilingual Matters.

Byram, M. (2019). Evaluation/Assessment und Selbstevaluation/Assessment interkultureller Kompetenzen. In C. Fäcke \& F.-J. Meißner (Eds.), Handbuch Mehrsprachigkeits- und Mehrkulturalitätsdidaktik (pp. 251-256).

Chilla, S., \& Vogt, K. (2017). Einleitung. In S. Chilla \& K. Vogt (Eds.), Heterogenität und Diversität im Englischunterricht: Fachdidaktische Perspektiven (pp.712). Frankfurt am Main: Peter Lang.

Council of Europe (2001). Common European framework of reference for languages: Learning, teaching, assessment. Retrieved from https://rm.coe.int/1680459f97

Council of Europe (2018). Common European framework of reference for languages. Learning, teaching, assessment: Companion volume with new descriptors. Retrieved from https://rm.coe.int/1680787989

Dausend, H. (2014). Mit Street Art transkulturelle Lernprozesse intiieren. In F. Matz, M. Rogge, \& S. Siepmann (Eds.), Transkulturelles Lernen im Fremdsprachenunterricht: Theorie und Praxis (1st ed., pp. 89-100). Frankfurt a.M: Peter Lang $\mathrm{GmbH}$ Internationaler Verlag der Wissenschaften.

Delanoy, W. (2014a). Mehrsprachigkeit, Englisch und Literatur(unterricht). Zeitschrift Für Interkulturellen Fremdsprachenunterricht, 19(1), 63-76.

Kapelari, Suzanne (Hg.), Vierte „Tagung der Fachdidaktik“ 2019:

„Interdisziplinäre fachdidaktische Diskurse zur Bildung für nachhaltige Entwicklung“

(C) 2020 innsbruck university press, ISBN 978-3-99106-019-2, DOI 10.15203/99106-019-2 
Delanoy, W. (2014b). Transkulturalität als begriffliche und konzeptuelle Herausforderung an die Fremdsprachendidaktik. In F. Matz, M. Rogge, \& S. Siepmann (Eds.), Transkulturelles Lernen im Fremdsprachenunterricht: Theorie und Praxis (1st ed., pp. 19-35). Frankfurt a.M: Peter Lang GmbH Internationaler Verlag der Wissenschaften.

Doff, S. (2016). Heterogenität im Fremdsprachenuntericht: Kontext - Aufbau und Inhalt - Ausgangs- und Kristallisationsounkte. In S. Doff (Ed.), Heterogenität im Fremdsprachenunterricht: Impulse-Rahmenbedingungen-Kernfragen-Perspektiven (pp. 1-5). Tübingen: Narr.

Euler, D. (2014). Design Research - a paradigm under development. In D. Euler \& S. F.E. Sloane (Eds.), Design-Based Research (pp. 15-44). Stuttgart: Franz Steiner Verlag.

Fäcke, C., \& Meißner, F. J. (2019). Einleitung. In C. Fäcke \& F.-J. Meißner (Eds.), Handbuch Mehrsprachigkeits- und Mehrkulturalitätsdidaktik (pp. 1-16).

Freitag-Hild, B. (2018). Teaching Culture - Intercultural Competence, Transcultural Learning, Global Education. In C. Surkamp \& B. Viebrock (Eds.), Teaching English as a Foreign Language: An Introduction (pp. 159-175). Stuttgart: J.B. Metzler.

Göbel, K., Lewandowska, Z. M.， \& Diehr, B. (2017). Lernziel interkulturelle Kompetenz - Lernangebote im Englischunterricht der Klassenstufe 9 - Eine Reanalyse der Unterrichtsvideos der DESI-Studie. Zeitschrift Für Interkulturellen Fremdsprachenunterricht, 22(1), 107-121.

Gogolin, I. (2016). Folgen der Migration für Bildung und Erziehung. In E. BurwitzMelzer, G. Mehlhorn, C. Riemer, K.-R. Bausch, \& H.-J. Krumm (Eds.), UTB: Vol. 8043. Handbuch Fremdsprachenunterricht (6th ed., pp.60-64). Tübingen: A. Francke Verlag.

Grünewald, A. (2012). Förderung interkultureller Kompetenz durch Lernaufgaben. Fremdsprachen Lehren Und Lernen, 41(1), 54-71.

Hu, A., \& Byram, M. (2009). Introduction. In A. Hu \& M. Byram (Eds.), Interkulturelle Kompetenz und fremdsprachliches Lernen: Modelle, Empirie, Evaluation = Intercultural competence and foreign language learning; models, empiricism, assessment (pp. VII-XXV). Tübingen: Gunter Narr Verlag.

Kramsch, C. (2006). Context and culture in language teaching ([Nachdr.]). Oxford applied linguistics. Oxford: Oxford Univ. Press.

Kuckartz, U. (2014). Mixed Methods: Methodologie, Forschungsdesigns und Analyseverfahren. Wiesbaden: Springer VS. https://doi.org/10.1007/978-3-531-93267-5 
Lo Bianco, J. (2009). Dilemmas of efficiency, identity and worldmindedness. In J. Miller, A. Kostogriz, \& M. Gearon (Eds.), New perspectives on language and education. Culturally and linguistically diverse classrooms: new dilemmas for teachers (pp. 113-131). Bristol, UK: Multilingual Matters.

Reimann, D. (2015). Inter- und transkulturelle kommunikative Kompetenz.

Reimann, D. (2017). Interkulturelle Kompetenz. Narr Starter. Tübingen: Narr Francke Attempto.

Schäfer, L. (2016). Wie kann kulturelles Lernen im Englischunterricht der Sekundarstufe I in heterogenen Lerngruppen angebahnt werden?: Erste Ergebnisse einer Design-Based Research-Studie. In S. Doff (Ed.), Heterogenität im Fremdsprachenunterricht: Impulse-Rahmenbedingungen-Kernfragen-Perspektiven (pp. 91-106). Tübingen: Narr.

Schäfer, L. (2017a). Förderung kulturell-visueller Kompetenzen mit Street Art im Englischunterricht. In S. Doff \& R. Komoss (Eds.), Making Change Happen: Wandel im Fachunterricht analysieren und gestalten (pp. 69-74). Wiesbaden: Springer VS.

Schäfer, L. (2017b). Von bunten Wänden und blumenwerfenden Demonstranten - Die Design-basierte Entwicklung und Erprobung einer Unterrichtssequenz zu Street Art im inklusiven Englischunterricht der Sekundarstufe I. In C. Schlaak \& S. Thiele (Eds.), Romanische Sprachen und ihre Didaktik: Band 62. Migration, Mehrsprachigkeit und Inklusion: Strategien für den schulischen Unterricht und die Hochschullehre (pp. 141-157). Stuttgart: ibidem-Verlag.

Schlaak, C. (2015). Fremdsprachendidaktik und Inklusionspädagogik: Herausforderungen im Kontext von Migration und Mehrsprachigkeit. Stuttgart: ibidem.

Statistik Austria (2018a). Bevölkerung 1951 bis 2016 nach demographischen Merkmalen. Retrieved from https://www.statistik.at/web de/statistiken/menschen und gesellschaft/bevoel kerung/volkszaehlungen registerzaehlungen abgestimmte erwerbsstatistik/be voelkerung nach demographischen merkmalen/078573.html

Statistik Austria (2018b). Schülerinnen und Schüler mit nicht-deutscher Umgangssprache im Schuljahr 2017/18. Retrieved from https://www.statistik.at/web de/statistiken/menschen und gesellschaft/bildung und kultur/formales bildungswesen/schulen schulbesuch/index.html

Statistik Austria (2019). Bevölkerung mit Migrationshintergrund seit 2008. Retrieved from https:/www.statistik.at/web de/statistiken/menschen und gesellschaft/bevoel kerung/bevoelkerungsstruktur/bevoelkerung_nach_migrationshintergrund/0694 43.html

Sturm, T. (2016). Lehrbuch Heterogenität in der Schule (2nd ed.). München: Ernst Reinhardt Verlag.

Kapelari, Suzanne (Hg.), Vierte „Tagung der Fachdidaktik“ 2019:

„Interdisziplinäre fachdidaktische Diskurse zur Bildung für nachhaltige Entwicklung“

(C) 2020 innsbruck university press, ISBN 978-3-99106-019-2, DOI 10.15203/99106-019-2 
Trautmann, M. (2010). Heterogenität. (k)ein Thema der Fremdsprachendidaktik? In A. Köker, S. Romahn, \& A. Textor (Eds.), Herausforderung Heterogenität: Ansätze und Weichenstellungen (pp. 52-64). Bad Heilbrunn: Klinkhardt.

UNESCO (2009). UNESCO world report: Investing in cultural diversity and intercultural dialogue. World reports series. Paris: Unesco.

UNESCO-Weltaktionsprogramm (2019). Bildung für nachhaltige Entwicklung: Bildung für nachhaltige Entwicklung, Inklusion, Migration und Demokratie-Lernen. Retrieved from https://www.bne-portal.de/de/einstieg/bildungsbereiche/schule\#

United Nations (2019a). Sustainable Development Goals. Retrieved from https://www.un.org/sustainabledevelopment/sustainable-development-goals/

United Nations (2019b). Sustainable Development Goals: Goal 10: Reduce inequality within and among countries. Retrieved from https://www.un.org/sustainabledevelopment/inequality/

United Nations (2019c). Sustainable Development Goals: Goal 4: Quality Education. Retrieved from https://www.un.org/sustainabledevelopment/education/

Welsch, W. (1999). Transculturality: The Puzzling form of Cultures Today. In M. Featherstone \& S. Lash (Eds.), Theory, culture \& society. Spaces of culture: City, nation, world (pp. 195-213). London, Thousand Oaks, Calif: Sage. https://doi.org/10.4135/9781446218723.n11

Wiek, A., Withycombe, L., \& Redman, C. L. (2011). Key competencies in sustainability: a reference framework for academic program development. Integrated Research System for Sustainability Science, 6(2), 203-218. https://doi.org/10.1007/s11625-011-0132-6 